\title{
Cisto aracnóide após trauma na coluna torácica com compressão medular: Relato de caso
}

\section{Posttraumatic Arachnoid Cyst in the Thoracic Spine with Medullary Compression: Case Report}

\author{
Igor de Barcellos Zanon ${ }^{1}$ Michel Kanas ${ }^{2}$ Marcos Augusto Stávale Joaquim² $^{2}$ M \\ Délio Eulálio Martins ${ }^{2(1)}$ Marcelo Wajchenberg ${ }^{2(1)}$ Nelson Astur ${ }^{1,2(0)}$
}

${ }^{1}$ Grupo de Coluna Vertebral, Departamento de Ortopedia e Traumatologia "Pavilhão Fernandinho Simonsen", Santa Casa de Misericórdia de São Paulo, São Paulo, SP, Brasil

${ }^{2}$ Departamento de Ortopedia e Traumatologia, Hospital Israelita Albert Einstein, São Paulo, SP, Brasil

Endereço para correspondência Igor de Barcellos Zanon, MD, Departamento de Ortopedia e Traumatologia, $2^{\circ}$ andar, Sala do Grupo de Afecções da Coluna Vertebral, Rua Dr. Cesário Motta Júnior, 112, Vila Buarque, São Paulo, SP, Brasil (e-mail: igorzanon@gmail.com).

Rev Bras Ortop 2021;56(1):114-117.

\section{Resumo \\ Palavras-chave \\ - cistos aracnóideos \\ - traumatismos da medula espinhal \\ - compressão da medula espinal}

\section{Abstract}

recebido

29 de Setembro de 2019

aceito

05 de Maio de 2020

Publicado online

Setembro 30, 2020
Cistos aracnóides são raros, podem ocorrer em todos os níveis do saco dural, e sua origem pode ser congênita, traumática, iatrogênica ou inflamatória. Neste artigo, relatamos o caso de uma paciente com mielopatia torácica compressiva decorrente de um cisto aracnóide intradural incomum, de manifestação pós-traumática, assim como sua resolução, além de realizar revisão da literatura sobre o tema. A principal localização é na coluna torácica, seguida das colunas lombar, lombossacra e toracolombar. O cisto com origem traumática é causado por lesão da camada interna da duramáter. Essas lesões produzem déficit neurológico por meio de efeito de massa sobre a medula espinhal. A existência de mielopatia compressiva associada é mais rara ainda. A ressecção ou drenagem dos cistos na vigência de mielopatia deve ser imediata, sendo o tratamento de escolha. Apesar de raros, podem ser uma complicação de fraturas da coluna vertebral que são muito comuns na prática de ortopedistas e neurocirurgiões, que devem estar preparados para essa situação incomum.

Arachnoid cysts are rare; they can occur at all levels of the dural sac, and can have a congenital, traumatic, iatrogenic or inflammatory origin. In the present article, we report a patient presenting a compressive thoracic myelopathy due to an unusual intradural arachnoid cyst with posttraumatic manifestation and its resolution, in addition to a literature review on the subject. These cysts mainly occur at the thoracic spine, followed by the lumbar, lumbosacral and thoracolumbar spines. Traumatic cysts are caused by an injury to the inner dural layer. These lesions produce neurological deficits through a mass effect on the spinal cord. Concomitant compressive
DOI https://doi.org/ $10.1055 / \mathrm{s}-0040-1714225$ ISSN 0102-3616.

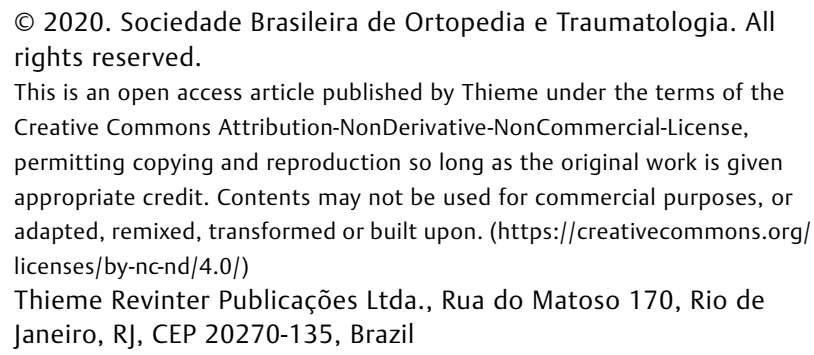

(c) 2020. Sociedade Brasileira de Ortopedia e Traumatologia. All rights reserved.

This is an open access article published by Thieme under the terms of the Creative Commons Attribution-NonDerivative-NonCommercial-License, permitting copying and reproduction so long as the original work is given appropriate credit. Contents may not be used for commercial purposes, or adapted, remixed, transformed or built upon. (https://creativecommons.org/ licenses/by-nc-nd/4.0/) Thieme Revinter Publicações Ltda., Rua do Matoso 170, Rio de Janeiro, RJ, CEP 20270-135, Brazil 
Keywords

- arachnoid cysts

- spinal cord injuries

- spinal cord compression myelopathy is even rarer. In case of myelopathy, cyst resection or drainage is the treatment of choice, and it must be performed immediately. Although rare, arachnoid cysts can be a complication of spine fractures; as such, orthopedists and neurosurgeons, who commonly see these injuries, must be prepared for this unusual situation.

\section{Introdução}

Cistos aracnóides são raros, podem ocorrer em todos os níveis do saco dural, e sua origem pode ser congênita, traumática, iatrogênica ou inflamatória. ${ }^{1,2}$ Acredita-se que a formação do cisto ocorra por herniação da membrana aracnóide por conta de falha na dura-máter. Os cistos traumáticos são ainda mais raros, e ocorrem por traumas penetrantes, fraturas da coluna vertebral com lesões durais por fragmentos ósseos, e lesões durais iatrogênicas no intraoperatório. ${ }^{3}$ Os locais mais comuns do óstio do cisto são: o ponto de entrada da raiz dorsal no saco dural ou na linha média. ${ }^{1}$ A apresentação clínica pode variar desde casos assintomáticos até aqueles em que os pacientes apresentam dor, fraqueza, parestesias e paralisia, por compressão neural decorrente. A avaliação por exames de imagem inclui tomografia computadorizada (TC), mielografia, e ressonância magnética (RM). Quando necessária, a intervenção cirúrgica consiste no fechamento primário do defeito na dura-máter, mediante abordagem posterior, com laminectomia e/ou costotransversectomia. ${ }^{1}$

Apesar de raros, os cistos aracnóides podem ser uma complicação de fraturas da coluna vertebral, que são muito comuns na prática de ortopedistas e neurocirurgiões, que, portanto, devem saber lidar eles.

Neste artigo, relatamos o caso de uma paciente com mielopatia torácica compressiva decorrente de um cisto de aracnóide intradural incomum, de manifestação pós-traumática, assim como sua resolução, além de realizar revisão da literatura sobre o tema.

\section{Relato de Caso}

Paciente do sexo feminino de 46 anos foi vítima de um acidente automobilístico havia 7 meses, com fratura por compressão da décima vértebra torácica do tipo B (A1) pela classificação do grupo Arbeitsgemainchaft für Osteosynthesefragen (AO, Comunidade de Trabalho para Problemas de Osteossíntese). ${ }^{4} \mathrm{Na}$ ocasião, foi realizado atendimento de urgência em outro hospital, e tratamento conservador da fratura com órtese tóraco-lombar. Após três meses, a paciente iniciou dificuldade de marcha com piora gradual, e, no último mês, evoluiu com paraparesia espástica acentuada e incapacidade para deambular. Ao procurar nosso serviço ambulatorial, a paciente apresentava força motora de grau III no membro inferior esquerdo e hipoestesia distal ao dermátomo L1. Os reflexos tendíneos profundos estavam exacerbados, com sinal de Babinski positivo e presença de clônus inesgotável. Os exames laboratoriais de rotina estavam dentro dos limites de normalidade. Radiografias da coluna torácica não evidenciaram alterações osteoarticulares agudas ou sequela da fratura. A RM evidenciou: fratura antiga do corpo vertebral de T10 com preservação da anatomia do corpo; alargamento do espaço entre os processos espinhosos de T9 e T10, sugerindo rotura ligamentar crônica; alteração do sinal da medula espinhal no nível de T9, T10 e T11, compatível com mielomalácia; e medula espinhal aderida posteriormente no nível de T7 a T9 e anteriormente de T9 a T12, associada a aracnoidite adesiva e cisto aracnóide ventral no canal vertebral nos níveis descritos, que deslocava posteriormente a medula (-Figura $\mathbf{1}$ ). A paciente foi submetida na mesma semana ao tratamento cirúrgico de descompressão com acesso por via posterior, laminectomia e costotransversectomia à esquerda em T9 e T10 para acesso anterior ao canal vertebral. Foi então realizada durotomia anterolateral com acesso direto ao cisto de aracnóide, drenagem e reparo do cisto, com náilon 5-0 e posterior estabilização, e artrodese com parafusos pediculares e hastes de T8 a T11 (-Figuras 2, 3 e 4). Não houve necessidade de órtese, e a reabilitação foi iniciada no primeiro dia de pós-operatório. A paciente evoluiu com melhora gradual do déficit neurológico em cerca de uma semana, e recuperação da força e da sensibilidade nos membros inferiores. Após seis meses, ela apresentou ataxia residual, mas deambulava sem necessidade de auxílio ou órtese. Não ocorreram complicações relacionadas ao sítio cirúrgico. Exames de controle evidenciaram posicionamento e alinhamento adequados do segmento fusionado, assim como consolidação óssea (- Figura 5).

\section{Discussão}

Cistos aracnóides são raros, e mais rara ainda é a existência de mielopatia compressiva associada. ${ }^{2,5,6}$ Os relatos da sua existência passaram a surgir com o uso mais frequente da RM. ${ }^{6} \mathrm{~A}$ maioria dos casos são de origem idiopática, e, muitas vezes, assintomáticos. ${ }^{5}$ Entre as possíveis etiologias, os casos póstraumáticos, como o apresentado neste artigo, são raros. A prevalência ainda não é um dado disponível na literatura, provavelmente por conta de os casos assintomáticos não despertarem interesse a ponto de serem publicados, sendo relatados apenas aqueles com mielopatia associada. ${ }^{7}$ A principal localização ocorre na coluna torácica, seguida das colunas lombar, lombossacra e toracolombar. ${ }^{8}$ Os cistos de origem traumática são causados por defeito da camada interna da dura-máter após trauma. ${ }^{5}$ Cistos ventrais podem causar fraqueza e mielopatia, enquanto os cistos dorsais, dor neuropática e parestesia. ${ }^{7}$ A diferença nos sintomas pode ser explicada porque as lesões ventrais podem produzir compressão na distribuição da artéria espinhal anterior e, assim, causar fraqueza ou mielopatia, enquanto os dorsais podem causar compressão local dos tratos espinhais, e, portanto, 


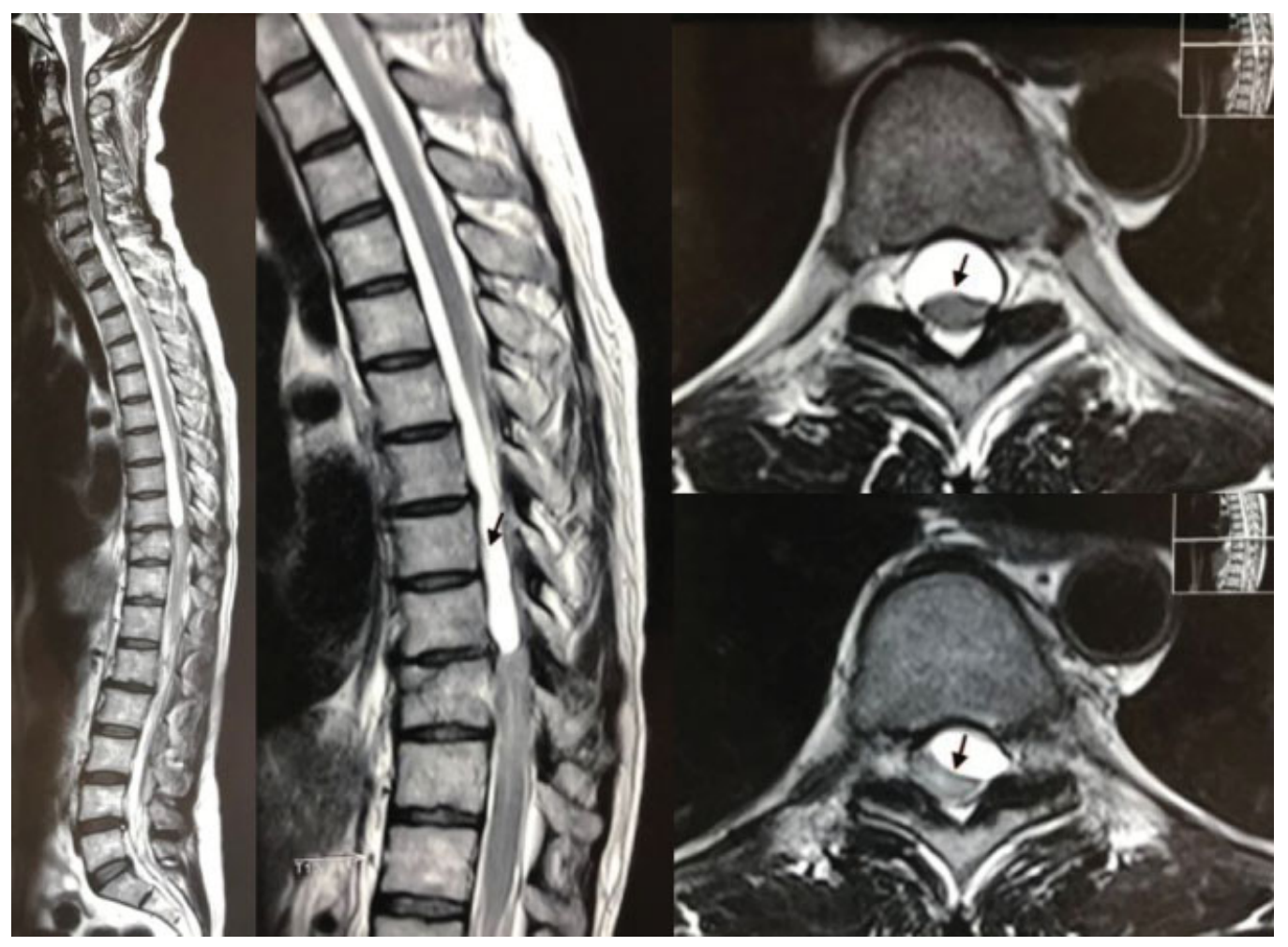

Fig. 1 Ressonância magnética, cortes sagitais, ponderada em T2, evidenciou: fratura antiga do corpo vertebral de T10; alargamento do espaço entre os processos espinhosos de T9 e T10, sugerindo rotura ligamentar crônica; alteração do sinal da medula espinhal no nível de T9, T10 e T11, compatível com mielomalácia; e medula espinhal aderida posteriormente no nível de T7 a T9 e anteriormente de T9 a T12, associada a aracnoidite adesiva e cisto aracnóide nos níveis descritos. Nos cortes axiais, no nível de T9, observamos a medula posteriorizada no canal vertebral e o cisto caracterizado pelo hipersinal. As setas em branco demonstram os limites do cisto e a aderência medular posterior.

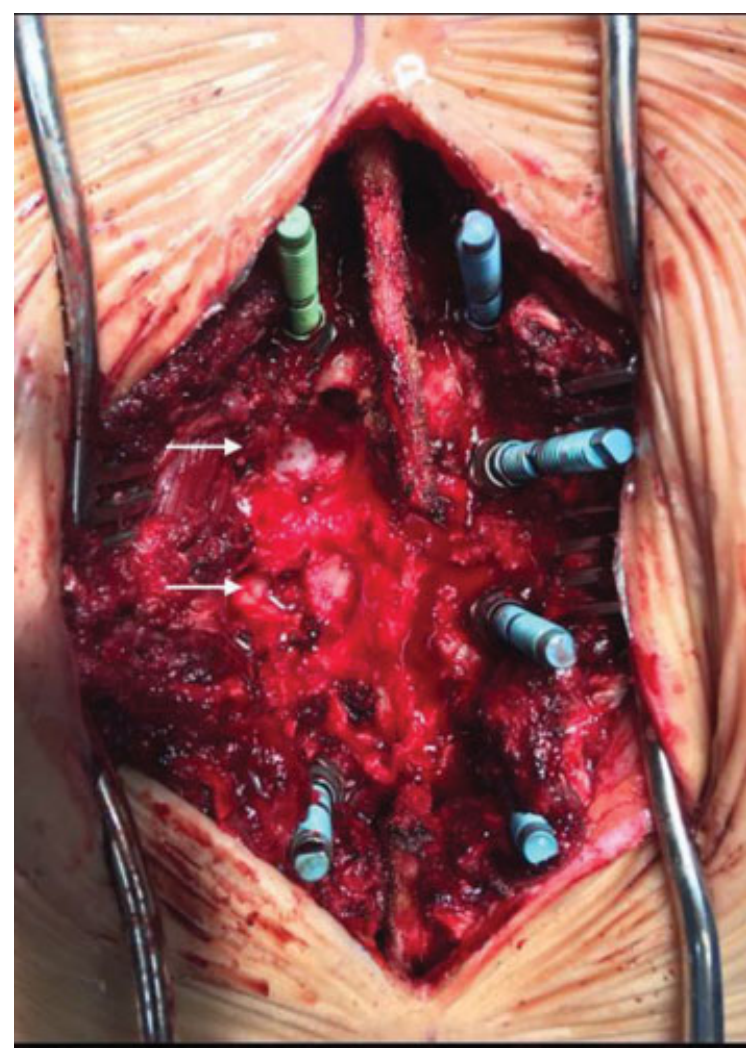

Fig. 2 Laminectomia e costotransversectomia (setas em branco) após a inserção de parafusos pediculares nos níveis de T8 e T11 para acesso ao cisto aracnóide e posterior estabilização.

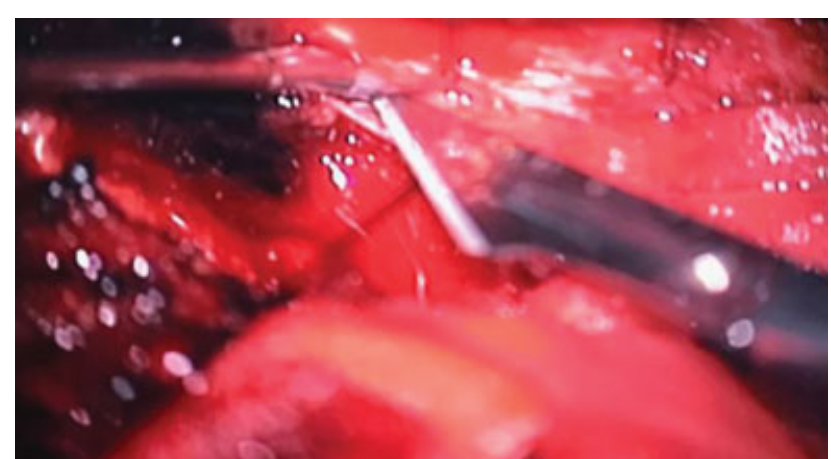

Fig. 3 Imagem do microscópio demonstrando a durotomia e a drenagem do cisto nos níveis T9 e T10.

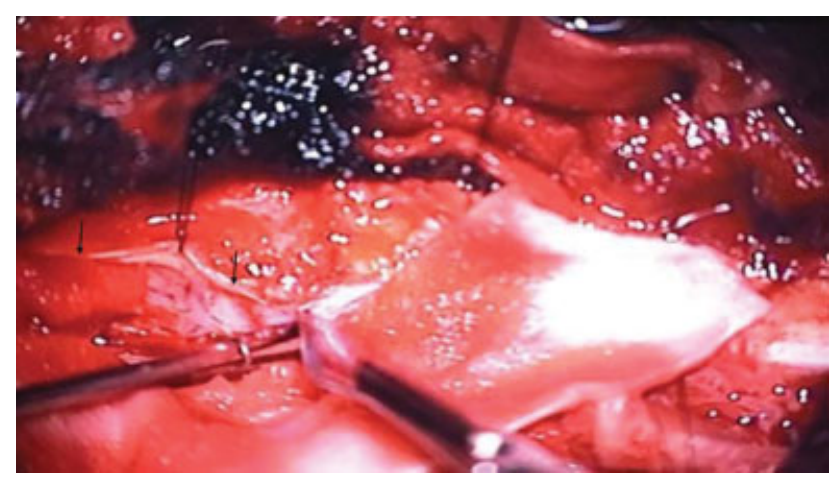

Fig. 4 Reparo do cisto aracnóide por meio de sutura primária com fios não absorvíveis de náilon 5-0. Borda da durotomia de acesso ao cisto em destaque nas setas. 


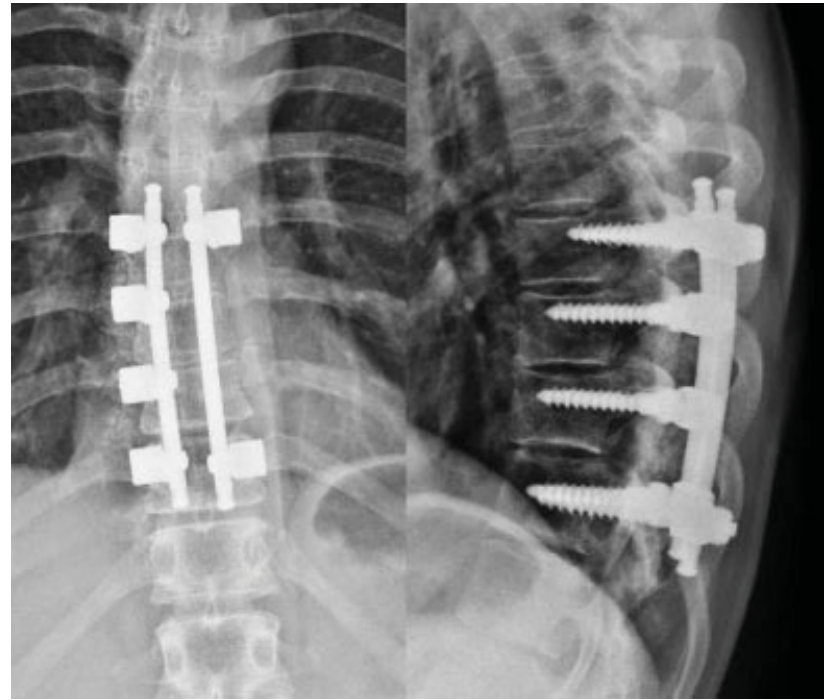

Fig. 5 Radiografias da coluna torácica, nas incidências posterolateral e de perfil, ao final do procedimento, demonstrando posicionamento e alinhamento adequados dos parafusos pediculares.

radiculopatia e dor. ${ }^{9}$ As recidivas podem ocorrer pela falha no tratamento da causa dos cistos; entretanto, a frequência de recorrência ainda é desconhecida. ${ }^{3}$ Os achados encontrados na mielografia e na TC são a compressão de estruturas adjacentes pelo cisto e o local de comunicação do cisto com o saco dural. $\mathrm{Na}$ RM, nota-se o efeito de massa das estruturas adjacentes e o hipersinal do fluido cerebroespinhal em imagens ponderadas em T2. ${ }^{1}$ A remoção radical dos cistos consiste no tratamento de escolha, mas, quando isso não é possível, por limitações técnicas, a drenagem do cisto deve ser realizada. ${ }^{4,6-10} \mathrm{~A}$ hemilaminectomia, com preservação do complexo ligamentar posterior, tem sido utilizada na maioria dos casos relatados na literatura, sendo suficiente para a abordagem do cisto, evitando complicações, como deformidade e instabilidade, e evitando a necessidade de estabilização adicional. No presente caso, a laminectomia foi associada a costotransversectomia em dois níveis devido à localização ventral do cisto e à necessidade de abordá-lo sem deslocar a medula. Podem ser utilizados fios não absorvíveis, e, em casos de falhas maiores, cobertura com gordura ou fáscia, substituto dural, ou selante de fibrina. ${ }^{1,2,5} \mathrm{Na}$ maioria dos relatos publicados, após a cirurgia, os pacientes evoluíram com melhora total do déficit neurológico e da mielopatia encontrados no momento do diagnóstico. ${ }^{1,3,7,9}$ Por se tratar de afecção rara, com poucas publicações, e de baixo nível de evidência, existe uma limitação para a reprodução na prática clínica e a discussão de possibilidades de tratamento.

Os cistos aracnóides são lesões raras, e podem produzir déficit neurológico por meio de efeito de massa sobre a medula espinhal. A ressecção ou drenagem dos cistos na vigência de mielopatia deve ser imediata, sendo o tratamento de escolha.

\section{Conflitos de Interesses}

Os autores declaram não haver conflito de interesses.

\section{Referências}

1 Yanni DS, Mammis A, Thaker NG, Goldstein IM. Traumatic fracture of thin pedicles secondary to extradural meningeal cyst. J Surg Tech Case Rep 2011;3(01):40-43

2 Shibata T, Nakamura H, Yamano Y. Intradural arachnoid cyst associated with thoracic spinal compression fracture: 7-year follow up after surgery. Spinal Cord 2001;39(11):599-601

3 Hernández-León O, Pérez-Nogueira FR, Corrales N. [Postraumatic epidural arachnoid spinal cyst: case report]. Neurocirugia (Astur) 2011;22(03):267-270

4 Vaccaro AR, Oner C, Kepler CK, et al. AOSpine Spinal Cord Injury \& Trauma Knowledge Forum. AOSpine thoracolumbar spine injury classification system: fracture description, neurological status, and key modifiers. Spine 2013;38(23):2028-2037

5 Chen HJ, Chen L. Traumatic interdural arachnoid cyst in the upper cervical spine. Case report. J Neurosurg 1996;85(02):351-353

6 Alvisi C, Cerisoli M, Giulioni M, Guerra L. Long-term results of surgically treated congenital intradural spinal arachnoid cysts. J Neurosurg 1987;67(03):333-335

7 Levin TL, Zimmerman RD, Lieberman H. Intradural spinal arachnoid cyst. Case report. Clin Imaging 1990;14(03):245-247

8 Kong WK, Cho KT, Hong SK. Spinal extradural arachnoid cyst: a case report. Korean J Spine 2013;10(01):32-34

9 Wang MY, Levi AD, Green BA. Intradural spinal arachnoid cysts in adults. Surg Neurol 2003;60(01):49-55, discussion 55-56

10 Kendall BE, Valentine AR, Keis B. Spinal arachnoid cysts: clinical and radiological correlation with prognosis. Neuroradiology 1982;22(05):225-234 